17. Messing, J. \& Vieira, J. Gene 19, 269-276 (1982).

18. Sanger, F., Nicklen, S. \& Coulson, A. R. Proc. natn. Acad. Sci. U.S.A. 74, 5463-5467 (1977)

19. Hafen, E., Levine, M., Garber, R. L. \& Gehring, W. J. EMBO J. 2, 617-623 (1983).

20. Angerer, L. M. \& Angerer, R. C. Nucleic Acids Res. 9, 2819-2840 (1981).

21. Krieg, P. A. \& Melton, D. A. Methods Enzym. 155, 397-415 (1987).

22. Clayton, D. F., Huecas, M. E., Sinclair-Thompson, E. Y., Nastiuk, K. L. \& Nottebohm, F. Neuron 1, 249-261 (1988).

ACKNOWLEDGEMENTS. This work was supported by the NHH. We are grateful to François Karch, Welcome Bender and Barbara Weiffenbach for showing us the unpublished sequence of abdA. We also thank Martin Chalfie and William McGinnis for their comments on the manuscript and Cen Zhang. Nick Necles, Helen Doyle, Chris Rushlow and Monica Driscoll for their help and advice.

\section{Fluorescence energy transfer shows that the four-way DNA junction is a right-handed cross of antiparallel molecules}

\author{
Alastair I. H. Murchie*, Robert M. Clegg†, \\ Eberhard von Kitzing $\dagger \neq$, Derek R. Duckett*, \\ Stephan Diekmann† \& David M. J. Lilley*§
}

* Department of Biochemistry, The University, Dundee DD1 4HN, UK $\uparrow$ Abteilung Molekulare Biologie, Max-Planck-Institut für Biophysikalische Chemie, D-3400 Göttingen, FRG

THE four-way junction between DNA helices is the central intermediate in recombination ${ }^{1-10}$, and the manner of its interaction with resolvase enzymes can determine the genetic outcome of the process. A knowledge of its structure is a prerequisite to understanding the interaction with proteins, and there has been recent progress $^{11-14}$. Here we use fluorescence energy transfer to determine the relative distances between the ends of a small DNA junction, and hence the path of the strands. Our results are consistent with the geometry of an ' $X$ '. The interconnected helices are juxtaposed so that the continuous strands of each helix generate an antiparallel alignment, and the two interchanged strands do not cross at the centre. The acute angle of the $X$ structure is defined by a right-handed rotation of the helical axes about the axis perpendicular to the $X$ plane, as viewed from the centre of the $X$.

The in vitro construction of defined DNA junctions ${ }^{11,15-17}$ has been essential to the understanding of the structure of the four-way junction. The structure introduces a sharp bend in the DNA $^{11}$. Using a technique related to that of Cooper and Hagerman $^{12}$, we studied the configuration of a series of junction ${ }^{13}$ and proposed a general model for the helical junctionthe stacked $X$ structure. The molecular geometry of the $X$ at the junction is formed by the pairwise stacking of the helical arms, resulting in quasi-continuous helices. Helix-helix stacking was first proposed by Sigal and Alberts ${ }^{18}$. The symmetry of our structure is consistent with results of hydroxyl radical probing of synthetic junctions ${ }^{14}$. The choice of stacking partners is governed by the sequence at the junction, and the pattern of cleavage by phage resolvase enzymes is dependent on the isomeric form adopted for a given DNA sequence ${ }^{13,19}$. In the absence of metal ions, junctions of any sequence adopt a structure close to square-planar, in which the four arms are unstacked and maximally extended.

Four stereochemical arrangements of the stacked $X$ structure are possible (Fig. 1). Gel electrophoresis experiments indicated that a non-crossed structure was correct ${ }^{13}$, but this conclusion rested on assumptions about the electrophoretic mobility of DNA. Here we have used an independent approach, employing fluorescence energy transfer techniques to measure relative endto-end distances in the junction. The stacked X-model predicts that two of the end-to-end distances from the possible six will

$\ddagger$ Present address: Max-Planck-Institut für Medizinizche Forschung, Abteilung Zellphysiologie, Postfach 103820, D-6900 Heidelberg. FRG. be shorter than the others, and identification of these will distinguish between crossed and non-crossed structures. Two fluorescent dyes attached to a macromolecule can exhibit an overlap of emission (donor) and excitation (acceptor) frequencies, when energy transfer then becomes possible from donor to acceptor. The transfer is dependent on the distance between the donor and acceptor (in the size range from 30 to $70 \AA$ ); the efficiency of transfer $(E)$ is given by $E \propto\left(1+\left(R / R_{0}\right)^{6}\right)^{-1}$, where $R$ is the distance between the dyes, and $R_{0}$ is the distance where the efficiency of transfer is 0.5 (refs 20,21 ).

We attached fluorescein and tetramethyl rhodamine to the $5^{\prime}$ ends of selected oligonucleotides used for the assembly of junctions to investigate the energy transfer across the six possible end-to-end distances, each of which could be studied in both directions. These experiments were carried out for a variety of arm lengths, but the best results were obtained using junctions constructed from oligonucleotides of 34 bases, giving junctions of arm length 17 base pairs (see below). As the strength of a dipole-dipole interaction is dependent on orientation as well as distance, at least one of the dyes had to be relatively mobile. Hence we used a $\mathrm{C}_{6}$ linker to attach the dye to the DNA. Subsequent measurements of fluorescence anisotropy indicated that there was some interaction between the dyes and the ends of the arms, so we included a $5^{\prime} \mathrm{CpC}$ on each arm to maintain a constant environment for the dyes. As we can unfold the junction by the removal of ions ${ }^{13}$, the energy transfer can be measured before and after the addition of magnesium while keeping all other factors constant.

The junction sequences chosen for these studies were essentially those of junctions 1 and 2 (ref. 13), previously shown to have different isomeric conformations. The central sequences are given in Fig. $2 a$. The ratios of fluorescence energy transfer for junctions 1 and 2 with and without the addition of $5 \mathrm{mM}$ magnesium ions are shown in Fig. $2 b$. For junction 2, the largest increase in energy transfer upon addition of $\mathrm{Mg}^{2+}$ was for the doubly labelled arms B-H or R-X. By contrast, the results for junction 1 , with the opposite isomeric configuration ${ }^{13}$, show the largest increase for the B-X and H-R labelled arms (Fig. 2b). Both these results verify the conclusions of our earlier gel electrophoresis experiments ${ }^{13}$. In addition, the relative energy

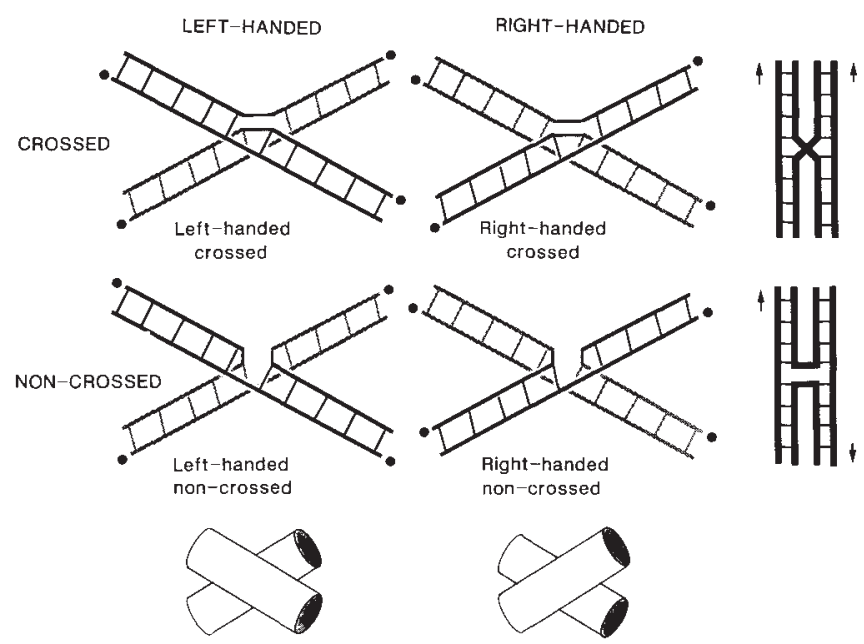

FIG. 1 Possible topology of strands and helices in the stacked X structure of the four-way junction. The strands can be drawn as non-crossed or crossed, generating an antiparallel or parallel alignment of the sequences respectively (indicated by the arrows). Because of the non-planarity of the $X$, the helices can cross in either a right-handed or a left-handed form (the convention for the handedness indicated is based on the definition of dihedral angles ${ }^{24}$ ). The helices at the back are stippled. The combination of these variables generates four possible structures. The $5^{\prime}$ termini of strands are indicated by filled circles, and are designated in such a way that all interconversions can be carried out in the plane of the page. 
transfer in the presence of $\mathrm{Mg}^{2+}$ between all the doubly labelled permutations of the derivatized junction 1 , and similarly for junction 2, corroborate these results. The relative energy transfer, and its increase by $\mathrm{Mg}^{2+}$, are consistent with only the noncrossed structure. Using estimates of the optical parameters of the dyes, we can calculate the distance between the shortest ends of the stacked X to be $\sim 1.5 R_{0}$. Using $R_{0}$ values from the literature $^{22}$, this gives $R=55-65 \AA$, which is a reasonable estimate if the acute angle is $60^{\circ}$. We stress, however, that this calculation includes several parameters that are not yet accurately determined for our experimental conditions, and therefore is subject to error. The strength of our conclusions about the stereochemistry of the four-way junction rests upon the comparison of the extent of energy transfer within a large group of nearly identical molecules, not upon a calculated value of $R$. The energy transfer experiments thus confirm all the details of our proposed model for the DNA junction. The observation of just two short distances is consistent with the $\mathrm{X}$ shape, and in the absence of cation these distances lengthen. The stacking partners are dependent on the sequence at the centre of the junction, exactly as we have described, and, most important,

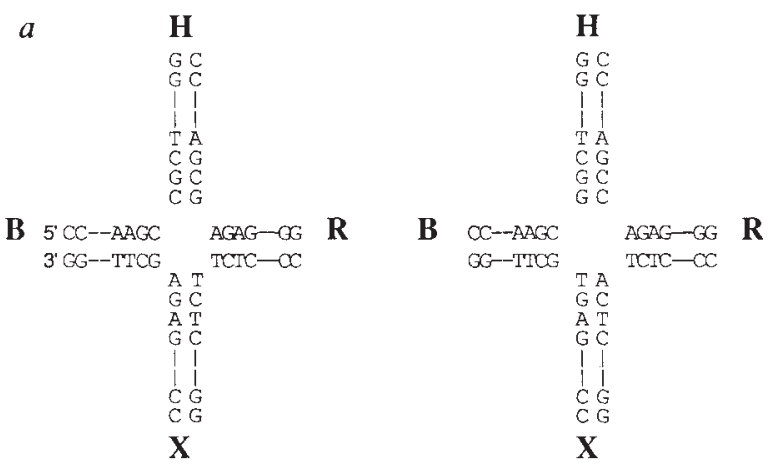

JUNCTION 1

JUNCTION 2 our results confirm that the $\mathrm{X}$ structure approximates most closely to the non-crossed, or anti-parallel, structure.

Can these results be translated into a structure that is stereochemically reasonable? Exploration of the four potential $\mathrm{X}$ structures (Fig. 1) using DNA models shows one arrangement to be particularly satisfactory, in that it avoids clashes between the deoxyribophosphate backbones in the vicinity of the point of joining. This is the right-handed cross, with non-crossed strands (Fig. 3). If the two long arms of the $X$ are orientated at $\sim 60^{\circ}$ to each other, the strands of one helix are aligned with the grooves of the other, and vice versa. Any other arrangement of the helices leads to unfavourable clashes between the backbones.

The stereochemical analysis predicts that the stacked X structure should be right-handed. Inspection of the right-handed, non-crossed model for junction 2 reveals that the phosphate situated 18 bases from the junction on the $5^{\prime}$ strand of arm B is closest to the $\mathrm{H}$ arm. We therefore prepared a series of labelled junctions in which fluorescein was attached to this position on arm B. In this series, we varied the length of the $\mathrm{H}$ arm (while maintaining the $5^{\prime} \mathrm{CpC}$ sequence) between 12 and 21 base pairs,

$b$
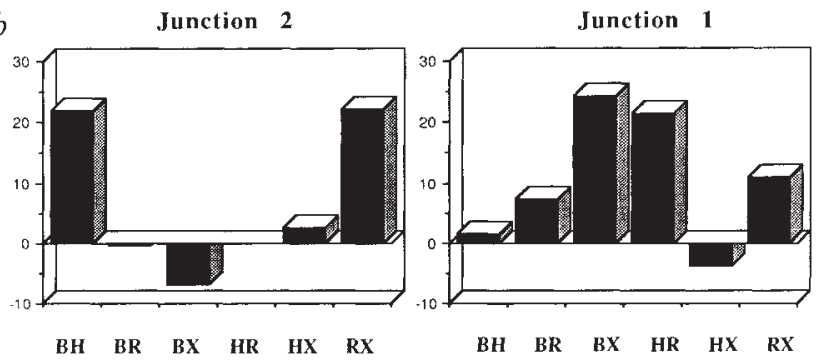

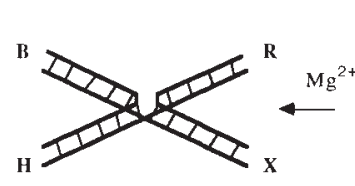

Junction 2

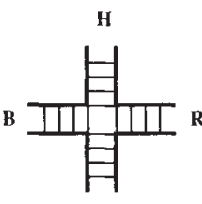

$\mathrm{X}$

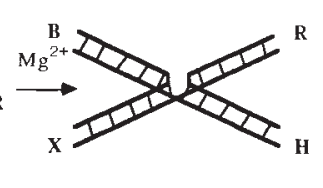

Junction 1
FIG. 2 Magnesium-induced changes in fluorescence energy transfer ratios measured for two junctions of different isomeric structure. a. Central sequences of the junctions used in these studies. The sequences of the central 15 base pairs of each arm were identical to those of junctions 1 and 2 used in gel electrophoresis experiments ${ }^{13}$. The arms are labelled $B, H, R$ and $X$. The junctions differ only in the reversal of two central base pairs, and previous experiments have indicated that this is sufficient to induce a change in stacking partners. Thus in junction 1, the B arm is stacked to form a co-linear helix with the $\mathrm{H}$ arm, whereas in junction 2 , the $\mathrm{B}$ and $\mathrm{X}$ arms are stacked ${ }^{13}$ The full sequences of the four oligonucleotides comprising junction 2 are CCTCCGTCCTAGCAAGCGGCTGCTACCGGAAGGG, CCCTTCCGGTAGCAGCCAGAGCGGTGGTTGAAGG, CCTTCAACCACCGCTCTACTCAACTGCAGTCTGG and CCAGACTGCAGTTGAGT. GCTTGCTAGGACGGAGG. $b$, Changes in fluorescent energy transfer on addition of magnesium ions. The emission spectra between 500 and $525 \mathrm{~nm}$ of the doubly labelled junctions are due only to the emission of the fluorescein label and thus the fluorescein contribution to the spectrum can be subtracted, leaving only a rhodamine spectrum containing emission which is due both to direct excitation and to energy transfer from fluorescein. The following values were then selected from the excitation spectrum and the fluorescein-subtracted emission spectrum: $F_{490 / 600}$ (emission at $600 \mathrm{~nm}$ with excitation at $490 \mathrm{~nm}$ ), contains both direct excitation $\left(F_{490 / 600}^{\text {dir }}\right)$ and energy transfer $\left(F_{490 / 600}^{\text {tran }}\right) ; F_{565 / 600}$, contains direct rhodamine excitation only. The ratio $R$ is thus measured

$R=F_{490 / 600} / F_{565 / 600}=\left(F_{490 / 600}^{\text {dir }}+F_{490 / 600}^{\text {tran }}\right) / F_{565 / 600}^{\text {dir }}=R_{565 / 600}^{490 / 600}+F_{490 / 600}^{\text {tran }} / F_{565 / 600}^{\text {dir }}$ The ratio $R_{565 / 600}^{490 / 600}$ is constant and only involves the absorption coefficients. As this is relatively small, $R$ measures the ratio of the fluorescence due to energy transfer to the direct fluorescence of rhodamine. These values were measured in the presence and absence of magnesium ions, and their difference is presented graphically. When this was measured for a doubly labelled isomer with both permutations of labels, an average is shown. As there is a linear relation between the absorption and fluorescence of rhodamine for all samples, this ratio normalizes the extent of transfer for all the samples, and the ratio is proportional to the per cent transfer. Only the optical constants of the dyes determine the proportionality constant. The schematic drawings below the graphs for junctions 2 and 1 illustrate the interpretation of the fluorescent energy transfer changes. For junction 2, the distances that shorten on magnesium-induced

folding, causing increased transfer, were $\mathrm{B}-\mathrm{H}$ and $\mathrm{R}-\mathrm{X}$, indicating a non-crossed structure with co-linear $B$ on $X$ and $H$ on $R$ arms. For junction 1 , the shortened distances are $\mathrm{B}-\mathrm{X}$ and $\mathrm{H}-\mathrm{R}$, indicating a non-crossed structure with co-linear $\mathrm{B}$ on $\mathrm{H}$ and $\mathrm{R}$ on $\mathrm{X}$ arms. METHODS. Preparation of dye-conjugated junctions: Oligonucleotides were synthesized on an Applied Biosystems 381A DNA synthesizer using $\beta$-cyanoethyl phosphoramidite chemistry $^{25.26} . \mathrm{AC}_{6}$ linker with a free amine group was incorporated at the $5^{\prime}$ end of the molecule by a final coupling with $N$-trifluoracetal-2-aminohexyl(2-methoxy)-NNdiisopropylamino phosphite ${ }^{27}$ (Applied Biosystems). Crude oligonucleotides were purified on an anion exchange column (Nucleogen, Diagen), followed by a $\mathrm{C}_{8}$ reversephase column (Aquapore, Applied Biosystems). Each oligonucleotide (one absorbance unit at $258 \mathrm{~nm}$ ) was reacted with $3 \mu \mathrm{l}$ of $50 \mathrm{mg} \mathrm{ml}^{-1}$ of the $N$-hydroxy-succinimide ester of tetramethylrhodamine (Applied Biosystems) in dimethylsulphoxide with $0.2 \mathrm{M}$ carbonate buffer $(\mathrm{pH} 9.5)$ to give a total volume of $15 \mu \mathrm{l}$ for $4-12 \mathrm{~h}$ at room temperature in the dark, and, in a separate reaction, with $20 \mu \mathrm{l}$ of $10 \mathrm{mg} \mathrm{ml}^{-1}$ fluorescein isothiocyanate (Sigma) in dimethylformamide with $0.3 \mathrm{M}$ carbonate buffer $(\mathrm{pH} 9.5)$ for $4-12 \mathrm{~h}$ at room temperature in the dark. After separation of unreacted dyes on Sephadex G25, conjugated oligonucleotides were purified by electrophoresis in a $20 \%$ polyacrylamide gel containing $7 \mathrm{M}$ urea. In order to construct junctions, oligonucleotide solutions $(0.04$ absorbance units at $258 \mathrm{~nm}$ ) were hybridized in appropriate combinations in $2 \mathrm{mM}$ $\mathrm{MgCl}_{2}, 3 \times \mathrm{SSC}$ at $65^{\circ} \mathrm{C}$, and slowly cooled to $10^{\circ} \mathrm{C}$. Junctions were purified by electrophoresis in $8 \%$ polyacrylamide gels in $90 \mathrm{mM}$ Tris-borate $(\mathrm{pH} 8.3), 2 \mathrm{mM} \mathrm{MgCl}_{2}$, with circulating buffer. Junctions were excised, electroeluted and ethanol-precipitated. Fluorescence spectroscopy: Fluorescence spectra were recorded using an SLM 8000 S fluorimeter. Excitation and emission spectra were corrected for lamp variation and instrumentation factors, and polarization artefacts were eliminated using magic angle conditions. Data were acquired and analysed with a DEC-LSI/23 computer. Dye concentrations were generally between 50 and $150 \mathrm{nM}$; per cent labefling was estimated from absorption spectra to be constant at $100 \%$ for all the samples. The absorption spectra of fluorescein, rhodamine and DNA were linearly related among all the samples and the excitation spectrum maxima that were due only to directly excited rhodamine were linearly related to the corresponding absorption spectra. The assembled dyelabelled junctions were dissolved in $90 \mathrm{mM}$ Tris-borate (pH 8.3), $0.1 \mathrm{mM}$ EDTA, for spectroscopy; conditions were identical to those used in gel electrophoresis experiments ${ }^{13}$. Magnesium chloride was added from a concentrated stock solution. 
and attached rhodamine to the 5 ' ends. This has the effect, shown diagramatically in Fig. $4 a$, of 'walking' the rhodamine dye around the $\mathrm{H}$ arm helix. Consideration of the right-handed model suggests that the two dyes should be closest when the rhodamine is attached to base 16 , so energy transfer should exhibit a maximum for this junction. For a left-handed cross the situation is reversed, with these phosphates located on opposite sides of the helix. The results are presented graphically in Fig. $4 b$. The energy transfer exhibited a maximum when the rhodamine was attached to phosphates 15 to 16 , in agreement with the prediction based on the right-handed structure. We therefore conclude that the four-way junction is indeed a righthanded, non-crossed structure.

A number of biological implications follow from the structure we have deduced for the four-way junction. Geneticists are accustomed to drawing the Holliday junction with the homologous DNA molecules aligned in a parallel manner, but this does not seem to be the natural energetic preference of DNA. Accessory proteins may influence the structure of recombination intermediates in the cell, but if that is so, then we have identified an important role for them. Antiparallel Holliday junctions would have consequences for branch migration. The $60^{\circ}$ cross implies that the point of contact between homologous DNA molecules is limited to a very small region around the junction. Antiparallel junctions complicate synapsis between homologous sequences, but not fatally, given the long-range flexibility of DNA molecules. Antiparallel orientation might simplify certain site-specific recombination processes: for example, the topology of integration and excision of phage $\lambda$ becomes symmetrical in both directions if the junction is drawn in the non-crossed form ${ }^{23}$. As physical information is available
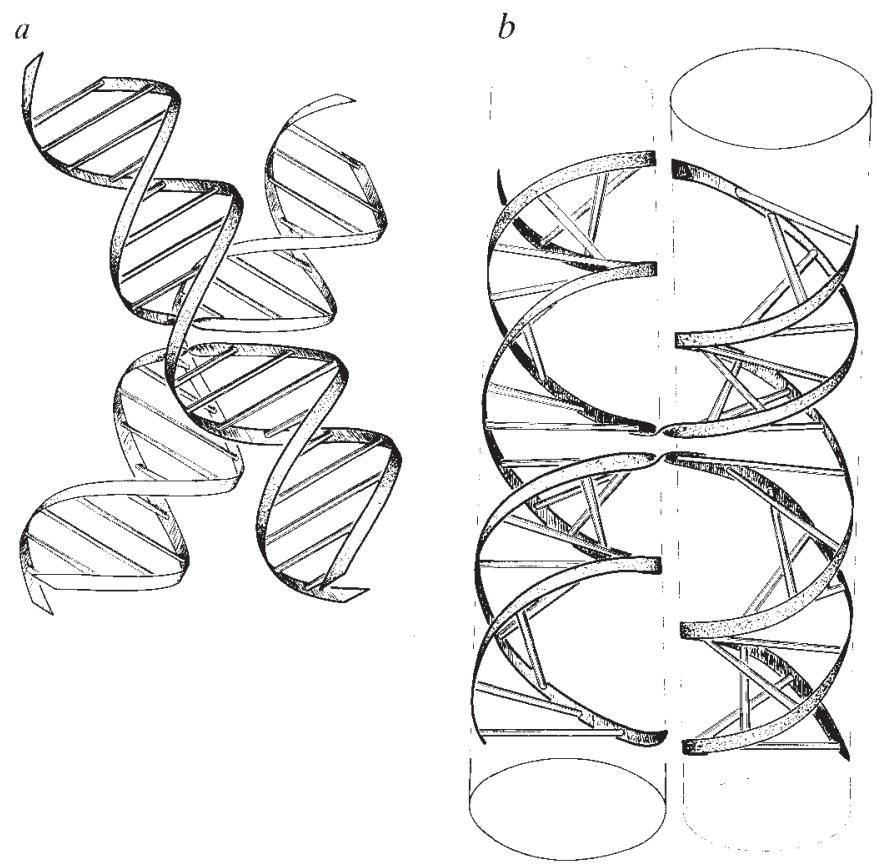

FIG. 3 A non-crossed, right-handed structure avoids unfavourable phosphate-phosphate interactions. $a$ and $b$, Front and side views of the structure. in this relative orientation of the two quasi-continuous helices, it can be seen that the continuous strands are accommodated in the major groove of the opposite helix, at the position closest to the centre of the junction. This point may be appreciated most readily by examination of two simple DNA models inclined correctly. Molecular mechanics calculations (E.v.K., D.M.J.L. and S.D., manuscript in preparation) indicate that the non-crossed right-handed structure does not violate stereochemical principles, and a related packing of helices has recently been found in a crystal of a doublestranded DNA oligonucleotide (D. Moras, personal communication).
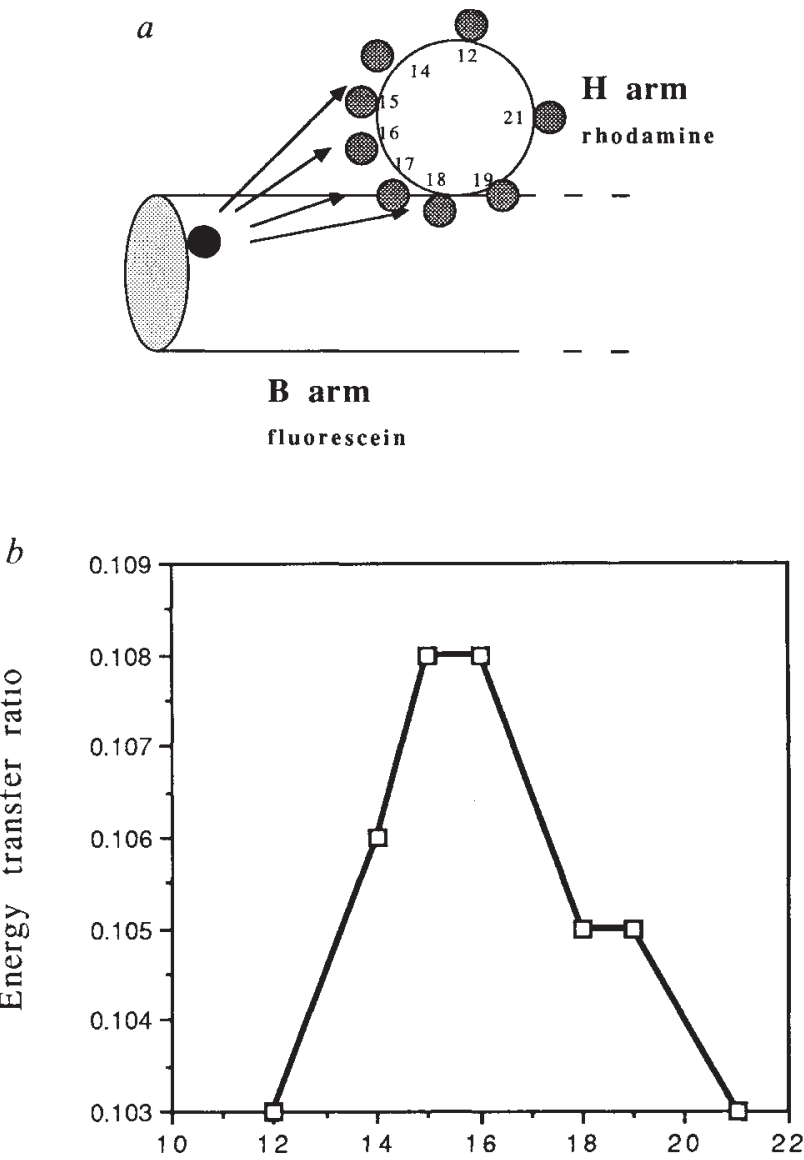

Length of $\mathrm{H}$-arm (bp)

FIG. 4 Fluorescence energy transfer indicates that the helices cross in a right-handed manner. Junctions based on the central sequence of junction 2 were constructed with arm lengths of $B$ (18 base pairs), $\mathrm{H}$ (12 to 21 base pairs), $R$ and $X$ (both 15 base pairs). These were prepared with dye-conjugated oligonucleotides as before, with fluorescein attached to the $5^{\prime}$ end of the $B$ arm, and rhodamine on the $\mathrm{H}$ arm. $\mathrm{a}$, Schematic to illustrate the relative dispositions of the $\mathrm{B}$ arm and $\mathrm{H}$ arm dyes for a right-handed cross. Model building indicates that the smallest dye-dye distance should occur when the $\mathrm{H}$ arm is $\sim 16$ base pairs. $b$, Energy transfer as a function of the position of rhodamine attachment. Plot is of fluorescence transfer ratio against the length of the $\mathrm{H}$ arm, for the junctions in the presence of $5 \mathrm{mM}$ magnesium ion. The transfer is clearly maximal for an $\mathrm{H}$-arm length of $15-16$ base pairs, in agreement with the prediction of a right-handed $X$ structure. Equivalent results were obtained (data not shown) for the reverse direction, $H_{\text {(fluorescein) }}-B_{\text {(rhodamine) }}$.

directly from fluorescence energy transfer experiments on nucleic acids, the technique might be applied profitably to related systems, such as the analysis of curved DNA in solution.

\footnotetext{
Received 27 June; accepted 14 September 1989.

1. Holliday, R. Genet. Res. 5, 282-304 (1964).

2. Meselson, M. S. \& Radding, C. M. Proc. natn. Acad. Sci. U.S.A. 72, 358-361 (1975)

3. Broker, T. R. \& Lehman, I. R. I molec. Biol. 60, 131-149 (1971)

4. Sobell, H. M. Proc natn. Acad Sci. U.S.A. 69, 2483-2487 (1972)

5. Sobell, H. M. in Mechanisms in Recombination (ed. Grell, R. F.) 433-438 (Plenum, New York, 1974)

6. Orr-Weaver, T. L., Szostak, J. W. \& Rothstein, R. J. Proc. natn. Acad. Sci. U.S.A. 78, 6354-6358 (1981).

7. Kitts, P. A. \& Nash, H. A. Nature 329, 346-348 (1987)

8. Nunes-Düby S. E Matsumoto, L \& Landy, A. Cell 50, 779-788 (1987).

9. Hoess, R., Wierzbicki, A. \& Abremski, K. Proc. natn. Acad. Sci. U.S.A. 84, 6840-6844 (1987).

10. Jayaram, M., Crain, K. L., Parsons, R. L. \& Harshey, R. M. Proc, natn Acad Sci. US. A. 85, 7902-7906 (1988).

11. Gough, G. W. \& Lilley, D. M. J. Nature 313, 154-156 (1985)

12. Cooper J. P. \& Hagerman. P. J \& molec. Biol 198, 711-719 (1987).
} 
13. Duckett, D. R. et al. Cell 55, 79-89 (1988)

14. Churchill, M. E. A.. Tullius, T. D., Kallenbach, N. R. \& Seeman, N. C. Proc. natn. Acad. Sci. U.S.A. 85, 4653-4646 (1988).

15. Bell, L. R. \& Byers, B. Proc. natn. Acad. Sci. U.S.A. 76, 3445-3449 (1979).

16. Kallenbach, N. R. Ma, R-1. \& Seeman N. C. Nature 305, 829-831 (1983).

17. Hsu, P. L. \& Landy, A. Nature 311, 721-726 (1984)

18. Sigal, N. \& Alberts, B. J. molec. Biol. 71, 789-793 (1972)

19. Mueller, J. E., Kemper, B., Cunningham, R. P., Kalenbach, N. R. \& Seeman, N. C. Proc. natn. Acad Sci. U.S.A. 85, 9441-9445 (1988).

20. Forster, T. Ann. Phys. 2, 55-75 (1948).

21. Förster, T. Fluorescence Organischer Verbindungen (Vandenhoeck \& Ruprecht, Göttingen, 1951)

22. Fairclough, R. H. \& Cantor, C. R. Meth. Enzym. 48, 347-379 (1978).

23. Stark, M., Sherratt, D. \& Boocock, M. Cel/ 58, 779-790 (1989)

24. Klyne, W. \& Prelog, V. Experientia 16, 521-523 (1960).

25. Sinha, N. D., Biernat, J., McManus, J. \& Koster. H. Nucleic Acids Res. 12, 4539-4557 (1984).

26. Beaucage, S. J. \& Caruthers, M. H. Tetrahedron Lett. 22, 1859-1862 (1981).

27. Coull, J., Wright, L. \& Bischoff, R. Tetrahedron Lett. 27, 3991-3995 (1986).

ACKNOWLEDGMENTS. We thank Annelies Zechel for skilled technical assistance, Tom Jovin for discussion, and the MRC, Wellcome Trust and Royal Society for financial support.

\section{CORRECTION}

\section{Correlation between the anaesthetic effect of halothane and saturable binding in brain}

\author{
A.S. Evers, B.A. Berkowitz \& D. A. d'Avignon
}

Nature 328, 157-160 (1987).

IN a previous issue of Nature, we reported that the anaesthetic effect of halothane correlated with the occupancy of a saturable chemical environment in rat brain ${ }^{1}$. We have extended these observations by examining the equilibration of halothane between cerebral cortical brain slices and anaesthetic-equilibrated buffer, an experimental paradigm in which whole-animal physiology cannot influence brain anaesthetic uptake. These studies showed that brain-slice halothane concentration was, with only minor deviation, a linear function of buffer concentration. This discrepancy between in vitro and in vivo equilibration studies led us to re-examine in vivo uptake of anaesthetic by simultaneously measuring brain and blood halothane concentrations in rats anaesthetized with various inspired concentrations of halothane. These studies showed higher brain and plasma halothane concentrations than those we originally reported (Fig. $1)$ : this results from improved calibration technique and possibly, in plasma, from reduced loss of halothane by volatilization during sample preparation. Brain and blood halothane concentrations were seen to rise in parallel as a function of inspired concentration, and the brain/blood halothane ratio (inset of Fig. 1) was constant over the entire inspired concentration range studied. The observed nonlinear relationship between inspired and blood halothane concentrations was not the result of blood saturation, as in vitro halothane/gas partition coefficients $\left(37^{\circ} \mathrm{C}\right)$ were the same $(2.78 \pm 0.08)$ at $1 \%(\mathrm{v} / \mathrm{v})$ and $3 \%$ halothane. These data indicate that anaesthetic-induced depression of halothane uptake into blood is predominantly responsible for the apparent saturation of brain observed in vivo; the apparent saturation reported earlier ${ }^{1}$ is not the result of a limited number of binding sites in brain.

We have also reported the existence of two chemical environments for halothane (and for other volatile anaesthetics) in brain $^{2}$, as defined by ${ }^{19} \mathrm{~F}$-NMR spin-spin relaxation behaviour ${ }^{1}$. The two environments are differentially occupied as a function of both inspired anaesthetic concentration and duration of

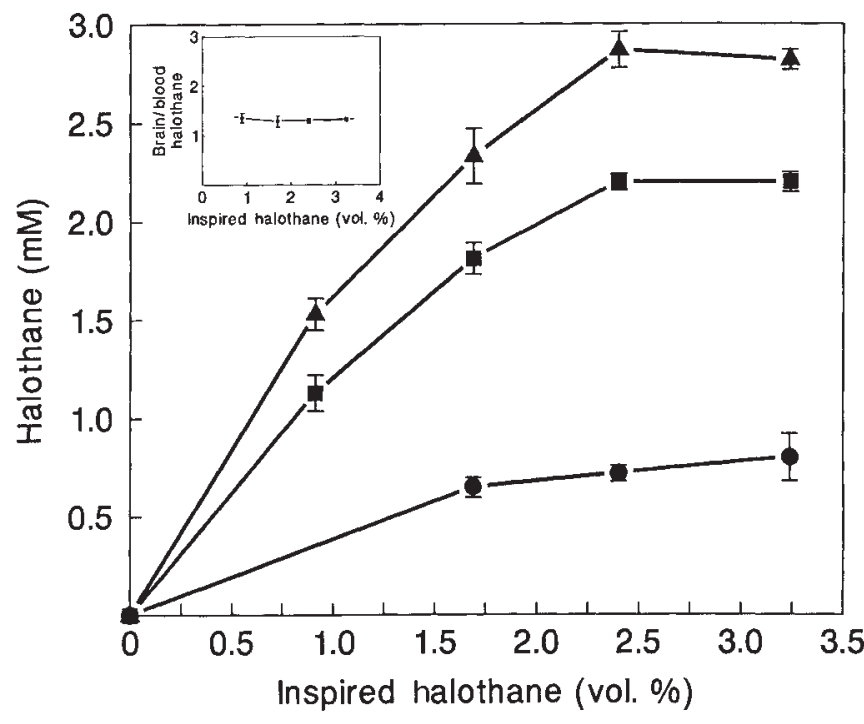

FIG. 1 Brain and blood halothane concentrations simultaneously measured in spontaneously ventilating rats. Rats were allowed to breathe various inspired concentrations of halothane (measured by gas chromatography) for 1 hour. Arterial blood was withdrawn and the animals were decapitated. Blood halothane concentrations ( $\square$ ) were measured by gas chromatography. and brain halothane concentrations (A) were measured both by gas chromatography and by NMR (ref. 1). Gas chromatography and NMR measurements of brain halothane gave identical results. In parallel experiments, plasma halothane concentrations (O) were measured by gas chromatography. Note that plasma halothane concentrations were reasonably well predicted by our in vitro measurement of the rat plasma/gas partition coefficient of $\sim 1$. The inset shows the brain/blood halothane concentration ratio plotted as a function of inspired concentration. All data are the mean \pm s.d. of 3-6 determinations.

delivery; occupancy of one of these environments (that with a short $T_{2}$ ) correlates with the anaesthetic effect of halothane. In light of our recent findings, it is apparent that occupancy by halothane of the short $T_{2}$ environment scales with total brain concentration; this environment is not saturable over the range of concentrations attainable in vivo. The other (long $T_{2}$ ) halothane environment represents an increasing proportion of total brain halothane concentration as a function of increasing inspired concentration and duration of anaesthetic administration. The basis for differential occupancy of the two halothane environments is not known, but could be the result of time- and concentration-dependence of either anaesthetic-induced membrane disruption ${ }^{3}$ (producing the long $T_{2}$ ) or changes in regional blood-flow distribution (for example, to white and grey matter). Although the physical chemical basis for the ${ }^{19} \mathrm{~F}-\mathrm{NMR}$ relaxation behaviour of anaesthetics in brain remains unknown, this relaxation behaviour is likely to be pharmacologically relevant, as evidenced by the strong correlation between volatile anaesthetic potency and ${ }^{19} \mathrm{~F}-\mathrm{NMR} T_{2}$ values in brain ${ }^{2}$. We are continuing to investigate the structural basis for and the pharmacological relevance of anaesthetic ${ }^{19} \mathrm{~F}$ relaxation behaviour in brain. This letter is submitted to correct the record as regards our previous report ${ }^{1}$.

1. Evers, A. S., Berkowitz, B. A. \& D. A. d'Avignon Nature 328, 157-160 (1987)

2. Evers, A. S., Haycock, J. C. \& D. A. d'Avignon Biochem. Biophys. Res. Commun. 151, 1039-1045 (1988).

3. Rottenberg, B. Waring. A. \& E. Rubin Science 213, 583-589 (1981). 